\title{
Ultrabroadband Dispersive Radiation by Spatiotemporal Oscillation of Multimode Waves
}

\author{
Logan G. Wright, ${ }^{1, *}$ Stefan Wabnitz, ${ }^{2}$ Demetrios N. Christodoulides, ${ }^{3}$ and Frank W. Wise ${ }^{1}$ \\ ${ }^{1}$ School of Applied and Engineering Physics, Cornell University, Ithaca, New York 14853, USA \\ ${ }^{2}$ Dipartimento di Ingegneria dell'Informazione, Università degli Studi di Brescia, \\ and Istituto Nazionale di Ottica, CNR, via Branze 38, 25123 Brescia, Italy \\ ${ }^{3}$ CREOL, College of Optics and Photonics, University of Central Florida, Orlando, Florida 32816, USA
}

(Received 17 August 2015; published 25 November 2015)

\begin{abstract}
In nonlinear dynamical systems, qualitatively distinct phenomena occur depending continuously on the size of the bounded domain containing the system. For nonlinear waves, a multimode waveguide is a bounded three-dimensional domain, allowing observation of dynamics impossible in open settings. Here we study radiation emitted by bounded nonlinear waves: the spatiotemporal oscillations of solitons in multimode fiber generate multimode dispersive waves over an ultrabroadband spectral range. This work suggests routes to sources of coherent electromagnetic waves with unprecedented spectral range.
\end{abstract}

DOI: 10.1103/PhysRevLett.115.223902

PACS numbers: 42.65.Sf, 42.81.Dp, 42.65.Jx, 42.65.Ky

Eigenmodes are ubiquitous tools for describing complex wave systems. For nonlinear complex wave systems, however, the superposition principle is not applicable. In special cases, these systems possess solitons, which act to some extent as nonlinear eigenmodes. Combined with more general nonlinear attractors and insights from linearized systems, researchers may build up a conceptual understanding of complex nonlinear wave dynamics. In optics, one-dimensional (1D) dynamics in single-mode waveguides have been thoroughly explored, with many advances hinging on the robust nonlinear attraction of solitons [1-3]. In unbounded 3D systems, dynamics have been explained largely in terms of a nonlinearly attracting instability: spatial or spatiotemporal collapse [4]. In reality, the collapse singularity is avoided by higher-order effects, and the field eventually expands. In this regime, promising results have been obtained considering conical waves, which are the eigenmodes of the 3D linear wave equation $[5,6]$. Conical wave solutions to the nonlinear wave equation may provide deeper insight [7,8]. Given the advantages the concepts of solitons and collapse have provided for studying single-mode waveguides and free space filamentation, it is natural to seek similar concepts in multimode waveguides. Multimode waveguides include as limiting cases single mode fiber (SMF, 1D) and free-space (3D), so these new concepts, whether solitons, nonlinearly attracting instabilities, guided conical waves, or something else entirely, could help to conceptually unify nonlinear optical dynamics across dimensions. More broadly, owing to similar mathematical descriptions, optical soliton dynamics in multimode waveguides should correspond to related effects in a wide variety of systems, e.g., Bose-Einstein condensates [9]. Furthermore, the generation and interaction of dispersive waves with optical solitons in one dimension has yielded many inspiring analogies [10-12], e.g., to Cerenkov radiation and event horizons. MMFs will allow more powerful test beds for these phenomena, with more controllable properties and a variable, higher dimensionality compared to SMFs.

Solitons in SMF have been intensely researched, because they are relatively accessible both analytically and experimentally. Equally important, soliton dynamics are critical to telecommunications, mode-locked fiber lasers, and compact white-light sources with high spatial mode quality. Multimode fibers (MMFs) could provide major benefits for various applications, from spatial division multiplexing in communications $[13,14]$, to high-power, versatile fiber lasers and white-light sources [15]. Although wave propagation in MMF is still experimentally and theoretically challenging, recent theoretical advances [16-21] make it more accessible. From a scientific perspective, MMF is an ideal environment for studying spatiotemporal nonlinear dynamics. By judicious design, or by control of the initial excitation, researchers may control the spatiotemporal characteristics of dynamics, through variation of the effective dimensionality, the coupling between modes, or their individual dispersions.

These factors have motivated recent work on nonlinear optical waves in MMFs [15,19,20,22-26]. In particular, we observed that launching $\approx 200-\mathrm{nJ}$ and 500 -fs pulses at $1550 \mathrm{~nm}$ into a graded-index (GRIN) MMF produces remarkable visible light emission characterized by a series of spectral peaks with nonuniform spacing [15]. Numerical simulations confirm these peaks, and suggest that even more spectacular emission occurs at long wavelengths, where fused silica is effectively opaque (Fig. 1). These observations are puzzling: some mysterious nonlinear optical mechanism generates and links electromagnetic radiation over 2 orders of magnitude in wavelength (from $>50$ to $<0.5 \mu \mathrm{m}$ ).

Here we show that these remarkable spectral features correspond to dispersive waves generated by the spatiotemporal oscillation of multimode solitons. The process is inherently 3D, with spatiotemporally evolving nonlinear waves emitting spatiotemporally evolving dispersive waves. However, we show that insights from solitons of 


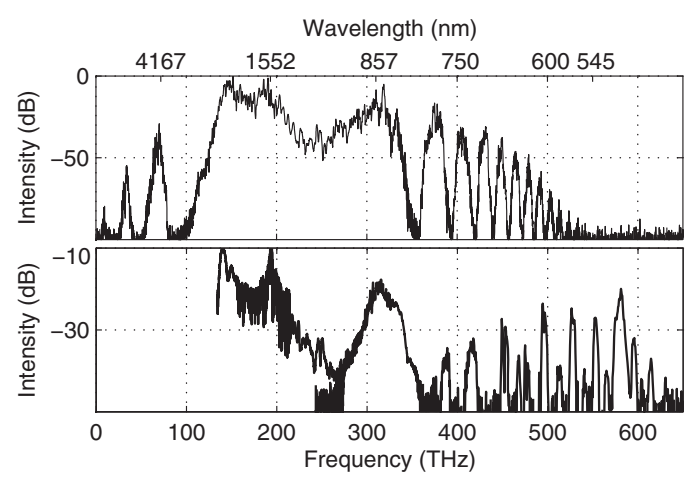

FIG. 1. Simulated (top) and example experimental (bottom) supercontinuum in multimode GRIN fiber. The pump pulse at $1550 \mathrm{~nm}$ creates a spectrum with a series of redshifted and blueshifted peaks. In the bottom panel, the $y$-axis reference $(0 \mathrm{~dB})$ is the maximum intensity of the $1550 \mathrm{~nm}$ pump peak.

the 1D nonlinear Schrödinger equation (NLSE), can prove useful in understanding the 3D system. This understanding suggests routes to generating ultrashort pulses in fiber at wavelengths outside current capabilities, reaching even outside the realm of optics.

Solitons are solutions to a conservative equation. To use them in applications such as lasers and telecommunications [27-38], loss must be compensated. This is accomplished by optical amplifiers in the cavity or transmission line. However, a periodic perturbation caused by gain and loss can destabilize a soliton. The origin of the instability, and the characteristic spectral sidebands that are its signature, is the fact that a periodic perturbation can phase match dispersive wave emission at particular frequencies $[39,40]$. For a perturbation period (spacing of amplifiers, or cavity length) $Z_{c}$, the phase-matching condition is approximately

$$
\left(k_{\mathrm{sol}}-k_{\mathrm{dis}}\right)=2 m \pi / Z_{c},
$$

where $m$ is an integer, and $k_{\mathrm{dis}}$ is the wave vector of the dispersive wave. $k_{\mathrm{sol}}$ is the soliton wave vector, which in the absence of higher-order dispersion is equal to $\left|\beta_{2}\right| / 2 \tau^{2}=$ $\pi /\left(4 Z_{0}\right)$, where $\beta_{2}$ is the group velocity dispersion, $\tau$ is the soliton duration, and $Z_{0}=\pi /\left(4 k_{\mathrm{sol}}\right)$ is the soliton period. This quasi-phase-matching leads to resonant emission at frequencies separated from the pump by

$$
\Omega_{\mathrm{res}}=\frac{1}{\tau} \sqrt{\frac{8 Z_{0}|m|}{Z_{c}}-1}
$$

$[36,40,41]$. This result was later refined to include the thirdorder dispersion of the fiber [42-44], where resonant frequencies were found to be roots of the equation

$$
\begin{aligned}
\left(k_{\mathrm{sol}}-k_{\mathrm{dis}}\right) & =2 m \pi / Z_{c} \\
& =\left(\frac{1}{2}+4 b_{3}^{2}-8 b_{3}^{4}\right)-\left(-\frac{\Omega^{2}}{2}-b_{3} \Omega^{3}\right),
\end{aligned}
$$

where $b_{3}=-\beta_{3} /\left(6\left|\beta_{2}\right| \tau\right)$ ( $\beta_{3}$ is the third-order dispersion) and $\Omega$ is the angular frequency separation from the pump (in units of $\tau^{-1}$ ). Production of dispersive waves is the primary limitation to the performance of soliton fiber lasers.

Although they were predicted in the 1980s [45-49], solitons consisting of pulses within multiple spatial modes have only recently been studied experimentally $[15,22,26]$. Initial work shows that solitary waves, termed MM solitons, can be excited in multimode graded-index (GRIN) fibers. Similar to 1D solitons, these pulses resist group-velocity dispersion and adjust adiabiatically due to perturbations such as Raman scattering. This behavior makes them a useful conceptual tool for understanding complex nonlinear processes that occur in supercontinuum generation in MMFs [15,26]. Unlike 1D solitons, MM solitons additionally resist modal velocity dispersion, i.e., the fact that each spatial mode in soliton has a different group velocity. Fission of MM solitons is spatiotemporal: it yields multiple MM solitons which can have many different modal distributions (spatiotemporal shapes). This spatiotemporal complexity makes their dynamics much richer than 1D solitons. While they are in some sense natural extensions of 1D NLSE solitons to MMF, they generally do not fulfill rigorous definitions of "soliton" (some exceptions are known $[19,20])$. Questions such as their longrange stability, how they interact with one another, and how many modes can be involved, remain largely unanswered.

When a beam excites multiple modes of a GRIN fiber, it propagates through the fiber with a characteristic spatial oscillation with period (pitch) $P=\pi R / \sqrt{2 \Delta}$, where $R$ is the core radius and $\Delta$ is the core-cladding relative index step $[22,50]$. This oscillation causes the intensity of the beam to periodically evolve, as in a loss-managed soliton transmission line or laser. For a pulsed beam, oscillations occur as long as the pulses in each mode maintain colocalization and a phase relationship [22]. Hence, a soliton containing multiple spatial modes experiences a periodic oscillation of its peak intensity and, therefore, is likely to emit resonant dispersive radiation.

We consider a simulation using the generalized multimode nonlinear Schrödinger equation (GMMNLSE) [16], with the first five radially symmetric modes excited uniformly for simplicity (Fig. 1). Experiments are conducted as described in Ref. [15]. In both simulation and experiment, we find that the soliton oscillation-induced dispersive waves (ODWs) are observed for many different initial spatial conditions, including without radial symmetry. Typically we observe more energetic ODWs with more intense initial conditions (large pulse energy, tight spatial localization, or both). Changing the initial noise level or the pulse duration also results in different dynamics. Nonetheless, provided sufficient pulse energy and fiber length, and that multiple modes are excited, the radiation's qualitative features (including spectral positions) are similar. In simulations, the energy of each ODW is distributed roughly equally among the modes, with the redshifted (blueshifted) sidebands exhibiting a slight preference for the low-order (higher-order) modes. 

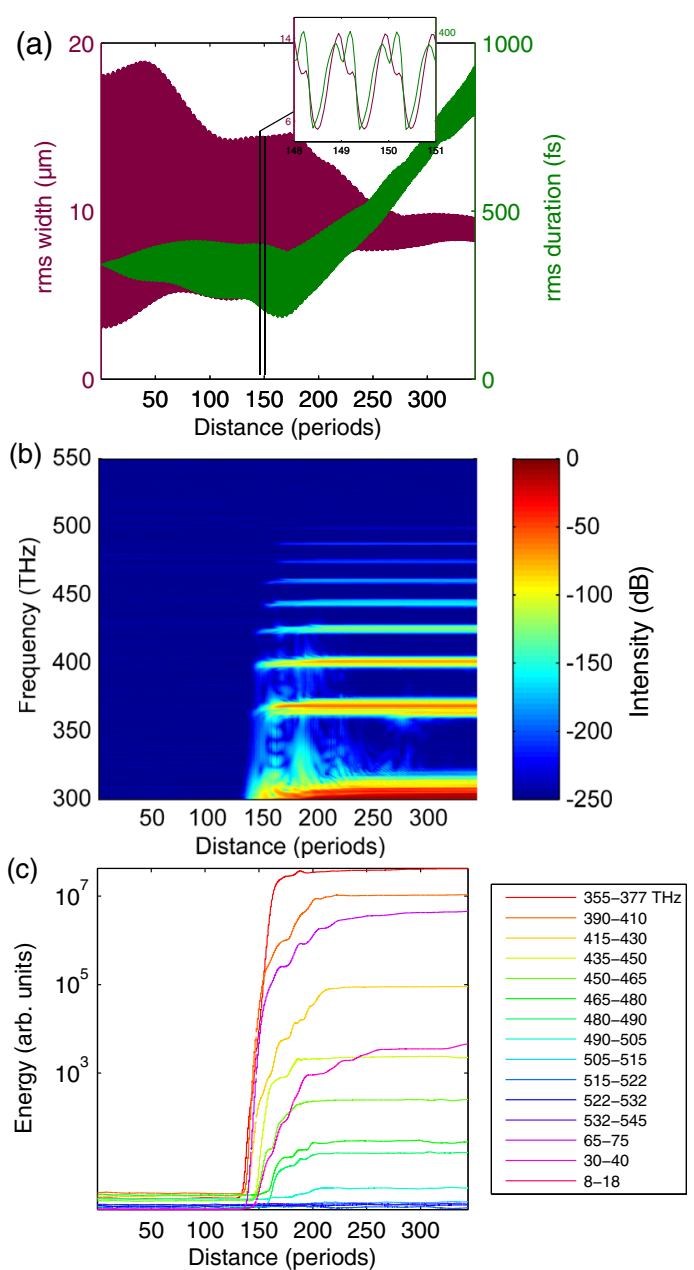

FIG. 2 (color online). Dynamics of dispersive wave formation in GRIN fiber. (a) Temporal and spatial breathing of the field (inset: zoom in near the onset of dispersive wave generation). (b) Evolution of the spectral intensity of the whole field through the same distance. (c) Energy in each dispersive wave band. The $x$-axis scales are normalized to the linear spatial oscillation period of the GRIN fiber, equal to $407 \mu \mathrm{m}$. These dynamics are also shown in Movies 1 and 2 of Supplemental Material [51], which provide a considerably more complete representation of the complex spatiotemporal evolution.

Representative simulation results for a $164-\mathrm{nJ}, 400-\mathrm{fs}$, 1550-nm MM pulse launched into a GRIN MMF are shown in Fig. 2. The results are also shown in Movies 1 and 2 of Supplemental Material [51]. The pulse's spatial and temporal breathing are evident in Fig. 2(a). Figure 2(b) shows the evolution of blueshifted ODWs [and Cerenkov radiation near $300 \mathrm{THz}(1000 \mathrm{~nm})]$. Redshifted ODWs are generated simultaneously. They are remarkably outside the transparency window of fused silica [the first appears at roughly $72 \mathrm{THz}(4200 \mathrm{~nm})$ ]. Attenuation is included in the simulations with an assumed frequency dependence $\alpha=\alpha_{1550} \exp \left\{-[f-c /(1550 \mathrm{~nm})] / b_{l}\right\}$, where $\alpha_{1550}=$ $0.05 \mathrm{~dB} / \mathrm{km}$ is the attenuation (units $\mathrm{m}^{-1}$ ) at $1550 \mathrm{~nm}$, and $b_{l}=0.0062 \mathrm{PHz}(\approx 80 \mathrm{~nm})$ models the increasing loss into the infrared. We neglect the increasing loss on the blue side of the spectrum. For these parameters, attenuation is $\approx 0.1 \mathrm{MdB} / \mathrm{km}$ at $4200 \mathrm{~nm}$. Despite this tremendous loss, redshifted and blueshifted ODWs have comparable energy. This is due to the relatively short length of the fiber (150 periods correspond to only $\approx 6 \mathrm{~cm}$ ), and to the enormous gain the ODWs experience: from the initial noise, the first blueshifted (redshifted) ODW exhibits $70 \mathrm{~dB}$ $(50 \mathrm{~dB})$ net gain in $1 \mathrm{~cm}$ [Fig. 2(c)]. After about 100 oscillation periods, the ODWs' energies saturate. The observed superexponential growth and subsequent saturation of the ODW energy is in qualitative agreement with the analytical prediction of the soliton perturbation theory that was developed to describe large-amplitude soliton intensity oscillations in periodically amplified transmission links $[52,53]$. On the other hand, for small amplitude oscillations of the soliton, the theory only predicts a linear growth of the ODW with distance [54].

To verify that the oscillations underlie the ODWs, we add oscillations artificially to a 1D NLSE by making the nonlinear coefficient a periodic function of the longitudinal coordinate,

$$
\begin{aligned}
\frac{\partial A(z, t)}{\partial z}= & -i \frac{\beta_{2}}{2} \frac{\partial^{2} A(z, t)}{\partial t^{2}}+\frac{\beta_{3}}{6} \frac{\partial^{3} A(z, t)}{\partial t^{3}} \\
& +i \gamma(z)|A(z, t)|^{2} A(z, t),
\end{aligned}
$$

where $A(z, t)$ is the pulse envelope, and $\gamma(z)$ is the $z$-dependent nonlinear coefficient. Figure 3 compares the result of solving this equation to the results above found using the GMMNLSE, and experiment. Figure 3(a) shows the solution of Eq. (4) with the indicated form of $\gamma(z)$. Because of the sinusoidal oscillation of the beam radius, one expects $\gamma(z)$ to be of the form $\gamma(z)=\gamma_{0} /\left\{\left[r_{\text {max }}-r_{\text {mean }}\right]+r_{\text {mean }} \sin (2 \pi z / P)\right\}^{2}$. We use the rms widths for $r_{\max }$ and $r_{\text {mean }}$ here, and rescale $\gamma_{0}$ appropriately, since simpler measures (e.g., mode-field diameter) are not well defined for the complex spatiotemporal fields that occur. At the onset of ODW generation $(\approx 145-155$ periods $), r_{\max }=14.5 \mu \mathrm{m}$ and $r_{\min }=5.2 \mu \mathrm{m}$, so that $\gamma(z)=\gamma_{0} /[9.8+4.6 \sin (2 \pi z / P)]^{2}$ [Typ. Oscillation, Fig. 3(b)]. The observed intensity oscillations of the MM field [GMMNLSE, Fig. 3(b)] are approximated better by $\gamma_{0} /[9.8+6.9 \sin (2 \pi z / P)]^{2}$ [Space + Time Fit, Fig. 3(b)]. This is because the MM soliton's duration also oscillates: solitons lengthen when their spatial width increases and shorten when their spatial width decreases [Fig. 2(a) and inset, and Movie 1 of the Supplemental Material [51]]. It is this spatiotemporal oscillation that generates the ODWs.

The experimental peak locations are consistent with simulation and analytic theory. Figure 3(c) shows the results of fitting the measured and simulated peak locations with the roots of Eq. (3) for various $m$. For the experimental (simulated) peaks, fitting yields $\beta_{2}=-26(-25) \mathrm{fs}^{2} / \mathrm{mm}$, and $\beta_{3}=118(143) \mathrm{fs}^{3} / \mathrm{mm}$. In both cases, the peaks are fit by the approximate model well. Given the uncertainties 

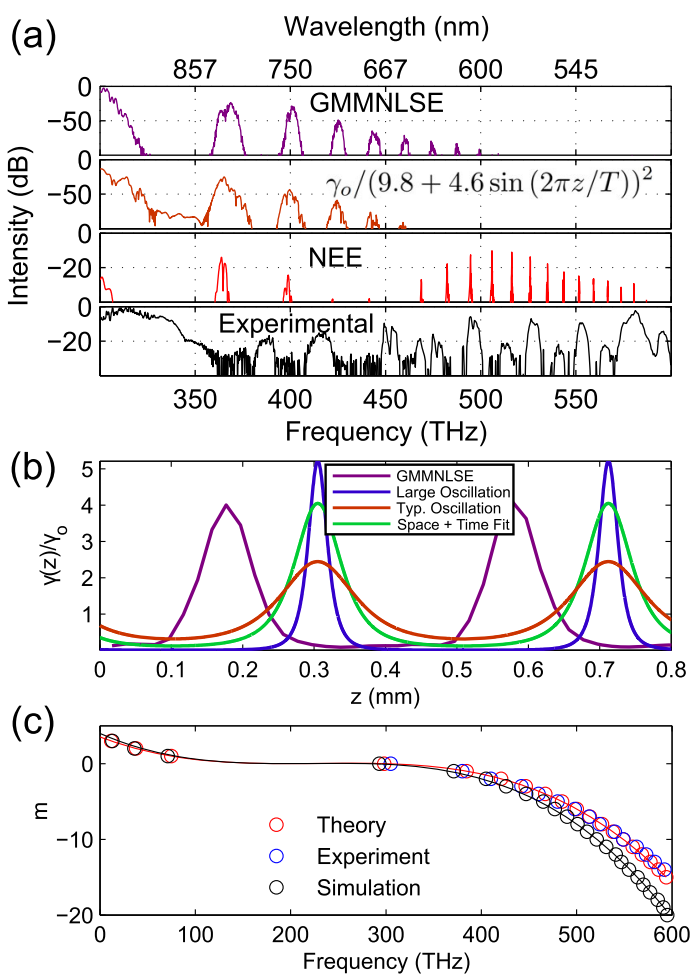

FIG. 3 (color online). (a) ODW from GMMNLSE simulations (GMMNLSE), Eq. (4) with $\gamma(z)$ as in text, 1D NEE simulations, and an experimental example spectrum. (b) Functions used in the quasi-1D approximation, along with the peak intensity variation of the field in GMMNLSE simulation. (c) Comparison of the periodic 1D phase-matching model with multimode simulations by the GMMNLSE and experiments in GRIN MMFs. Continuous curves are values of $m$ in Eq. (3), plotted with the best-fit parameter values.

on the experimental index profile, mode dispersions, and the modes involved in the oscillation, the agreement is excellent.

Although the measured ODW positions are well described, their amplitudes vary due to several effects beyond both the simulations and analytic model. First, we neglect all but 5 of the fiber's hundreds of guided modes. Currently, simulations with all the modes are impractical, and furthermore precise replication of experimental initial conditions is not possible. Neglected modes may allow phase matching of intramodal four-wave mixing (FWM) involving the 1550-nm pump, various dispersive waves, and Raman-shifted MM solitons. FWM could amplify redshifted ODWs at the expense of specific blueshifted ODWs. In the presence of many modes, intramodal FWM can be a complex process [55], particularly when cascades are considered [56]. Intramodal FWM is likely why, for certain initial spatial conditions, various low-order blueshifted ODWs are attenuated experimentally (several energy-conserving FWM processes exist for these spectral positions). We obtained the peak locations in Fig. 3(c) from experiments with multiple initial conditions, in order to account for ODWs attenuated in any particular condition. Second, because the spacing of the
ODWs is quite close to the Raman bandwidth of fused silica, certain ODWs may experience Raman gain from one another and from the third-harmonic light (TH). Last, the dynamic range and spectral resolution of the spectrometer limits the visibility of low-amplitude features, and broadens narrow spectral features.

Another remaining mystery is the relatively high amplitude of the ODWs observed in experiments, compared to simulations. Intramodal FWM may play some role, as well as larger oscillations and TH generation. Figure 3(a) shows the spectrum obtained by solving the 1D nonlinear envelope equation (NEE) with $\gamma(z)=\gamma_{0} /[15.5+14.5 \sin (2 \pi z / P)]^{2}$, [Large Oscillation, Fig. 3(b)], including self-steepening and assuming averaging of the oscillating Raman integral [57]. Larger oscillations produce relatively more intense dispersive waves, because the soliton is more strongly perturbed. Larger oscillations occur when more modes are coherently locked together [26], and as the experiment contains much more than 5 modes, we choose a functional form of $\gamma(z)$ to model this. The dynamics of TH generation are complex, but ultimately $\mathrm{TH}$ causes higher amplitudes at nearby wavelengths.

The ODWs generated in MMF have relevance to applications. For example, by filtering particular ODWs, one may generate pulses in wavelength regions well outside the gain spectrum of available fiber dopants. Tuning may be achieved by changing the pump wavelength or fiber pitch. The modulation instability gain spectrum of a $\mathrm{cw}$ field at the pump wavelength overlaps with the soliton sidebands [37]. Therefore, filtered ODWs could be parametrically amplified (by the circulating pump pulse or an injected cw field) if the chromatic walk-off between the pump and ODW fields was compensated. The inclusion of realistic loss in our simulations suggests that, remarkably, midinfrared radiation should be emitted from short silica fibers. However, future GRIN waveguides in chalcogenide or fluoride glasses, sapphire, or silicon would serve better. For the broadest operation, a hollow core, MM photonic crystal fiber would be the ultimate solution where spatiotemporal soliton oscillation may provide a means of generating mutually coherent, synchronized ultrashort pulses in different regions of the electromagnetic spectrum: e.g., a source of intense microwave, deep ultraviolet, and optical pulses.

In summary, we have shown that the spatiotemporal oscillation of nonlinear waves in GRIN multimode fiber causes the generation of spatiotemporal dispersive waves. These dispersive waves can be described relatively well by simulations using the GMMNLSE, and insight can be gained by approximating the dynamics with a quasi-1D model of longitudinally varying nonlinearity. Future work, involving more advanced models and experimental methods, can answer a few of the open mysteries about this process, the spatiotemporal dynamics leading to the multimode supercontinuum, and the spatiotemporal structure of the dispersive waves. This work provides a route to fiberbased ultrashort pulse sources with tunable wavelengths outside the range of any current fiber-optic technique. 
Portions of this work were funded by Office of Naval Research Grant No. N00014-13-1-0649. L. G. W acknowledges support from NSERC. S. W. is funded by Italian Ministry of University and Research (Grant No. 2012BFNWZ2). We thank C. Xu for loan of the laser used in the experiments and Alessandro Tonello for discussions on the relation of the work to BECs.

*lgw32@ cornell.edu

[1] A. Hasegawa, Appl. Phys. Lett. 23, 142 (1973).

[2] L. F. Mollenauer, R. H. Stolen, and J. P. Gordon, Phys. Rev. Lett. 45, 1095 (1980).

[3] Y. Kivshar and G. Agrawal, Optical Solitons: From Fibers to Photonic Crystals, 1st. ed. (Academic Press, New York, 2003).

[4] A. Couairon and A. Mysyrowicz, Phys. Rep. 441, 47 (2007).

[5] D. Faccio, M. A. Porras, A. Dubietis, F. Bragheri, A. Couairon, and P. Di Trapani, Phys. Rev. Lett. 96, 193901 (2006).

[6] D. Majus, G. Tamošauskas, I. Gražulevičiute, N. Garejev, A. Lotti, A. Couairon, D. Faccio, and A. Dubietis, Phys. Rev. Lett. 112, 193901 (2014).

[7] C. Conti, Phys. Rev. E 70, 046613 (2004).

[8] M. A. Porras, A. Parola, D. Faccio, A. Couairon, and P. Di Trapani, Phys. Rev. A 76, 011803 (2007).

[9] Y. S. Kivshar, T. J. Alexander, and S. K. Turitsyn, Phys. Lett. A 278, 225 (2001).

[10] N. Akhmediev and M. Karlsson, Phys. Rev. A 51, 2602 (1995).

[11] T. G. Philbin, C. Kuklewicz, S. Robertson, S. Hill, F. König, and U. Leonhardt, Science 319, 1367 (2008).

[12] R. Driben, A. V. Yulin, and A. Efimov, Opt. Express 23, 19112 (2015).

[13] P. J. Winzer, IEEE Photonics J. 4, 647 (2012).

[14] D. J. Richardson, J. M. Fini, and L.E. Nelson, Nat. Photonics 7, 354 (2013).

[15] L. G. Wright, D. N. Christodoulides, and F. W. Wise, Nat. Photonics, 9, 306. (2015).

[16] F. Poletti and P. Horak, J. Opt. Soc. Am. B 25, 1645 (2008).

[17] A. Mafi, J. Lightwave Technol. 30, 2803 (2012).

[18] J. Andreasen and M. Kolesik, Phys. Rev. E 86, 036706 (2012).

[19] A. Mecozzi, C. Antonelli, and M. Shtaif, Opt. Express 20, 23436 (2012).

[20] A. Mecozzi, C. Antonelli, and M. Shtaif, Opt. Express 20, 11673 (2012).

[21] S. Mumtaz, R.-J. Essiambre, and G. P. Agrawal, J. Lightwave Technol. 31, 398 (2013).

[22] W. H. Renninger and F. W. Wise, Nat. Commun. 4, 1719 (2013).

[23] P. S. J. Russell, P. Hölzer, W. Chang, A. Abdolvand, and J. C. Travers, Nat. Photonics 8, 278 (2014).

[24] F. Tani, J. C. Travers, and P. St. J. Russell, J. Opt. Soc. Am. B 31, 311 (2014).

[25] S. Buch and G. P. Agrawal, Opt. Lett. 40, 225 (2015).

[26] L. G. Wright, W. H. Renninger, D. N. Christodoulides, and F. W. Wise, Opt. Express 23, 3492 (2015).
[27] L.F. Mollenauer and R.H. Stolen, Opt. Lett. 9, 13 (1984).

[28] L. F. Mollenauer, R. H. Stolen, and M. N. Islam, Opt. Lett. 10, 229 (1985).

[29] H. Haus and M. Islam, IEEE J. Quantum Electron. 21, 1172 (1985).

[30] L. Mollenauer, J. Gordon, and M. Islam, IEEE J. Quantum Electron. 22, 157 (1986).

[31] Optical Solitons in Fibers, Springer Tracts in Modern Physics, edited by A. Hasegawa (Springer-Verlag, Berlin/ Heidelberg, 1989), Vol. 116.

[32] K. Smith, I. Armitage, R. Wyatt, N. Doran, and S. Kelly, Electron. Lett. 26, 1149 (1990).

[33] A. Ellis, J. Cox, D. Bird, J. Regnault, J. Wright, and W. Stallard, Electron. Lett. 27, 878 (1991).

[34] M. Nakazawa, E. Yoshida, and Y. Kimura, Appl. Phys. Lett. 59, 2073 (1991).

[35] D. Richardson, R. Laming, D. Payne, M. Phillips, and V. Matsas, Electron. Lett. 27, 730 (1991).

[36] J. P. Gordon, J. Opt. Soc. Am. B 9, 91 (1992).

[37] F. Matera, A. Mecozzi, M. Romagnoli, and M. Settembre, Opt. Lett. 18, 1499 (1993).

[38] H. A. Haus and W. S. Wong, Rev. Mod. Phys. 68, 423 (1996).

[39] N. Pandit, D. Noske, S. Kelly, and J. Taylor, Electron. Lett. 28, 455 (1992).

[40] S. Kelly, Electron. Lett. 28, 806 (1992).

[41] L. F. Mollenauer, K. Smith, J. P. Gordon, and C. R. Menyuk, Opt. Lett. 14, 1219 (1989).

[42] M. L. Dennis and I. N. Duling III, Appl. Phys. Lett. 62, 2911 (1993).

[43] M. L. Dennis and I. N. Duling III, Opt. Lett. 19, 1750 (1994).

[44] Y. Kodama, M. Romagnoli, S. Wabnitz, and M. Midrio, Opt. Lett. 19, 165 (1994).

[45] A. Hasegawa, Opt. Lett. 5, 416 (1980).

[46] B. Crosignani and P. D. Porto, Opt. Lett. 6, 329 (1981).

[47] B. Crosignani, A. Cutolo, and P. D. Porto, J. Opt. Soc. Am. 72, 1136 (1982).

[48] S.-S. Yu, C.-H. Chien, Y. Lai, and J. Wang, Opt. Commun. 119, 167 (1995).

[49] S. Raghavan and G. P. Agrawal, Opt. Commun. 180, 377 (2000).

[50] J. A. Buck, Fundamentals of Optical Fibers (John Wiley \& Sons, New York, 2004), p. 332.

[51] See Supplemental Material at http://link.aps.org/ supplemental/10.1103/PhysRevLett.115.223902 for discussion of the numerical model's assumptions and movies of pulse propagation.

[52] T. Georges and B. Charbonnier, Opt. Lett. 21, 1232 (1996).

[53] M. Midrio, M. Romagnoli, S. Wabnitz, and P. Franco, Opt. Lett. 21, 1351 (1996).

[54] J. N. Elgin and S. M. J. Kelly, Opt. Lett. 18, 787 (1993).

[55] M. Guasoni, Phys. Rev. A 92, 033849 (2015).

[56] J. Demas, P. Steinvurzel, B. Tai, L. Rishøj, Y. Chen, and S. Ramachandran, Optica 2, 14 (2015).

[57] G. Genty, P. Kinsler, B. Kibler, and J. M. Dudley, Opt. Express 15, 5382 (2007). 